\title{
Haar Wavelet Operational Matrix Method for Fractional Oscillation Equations
}

\author{
Umer Saeed and Mujeeb ur Rehman \\ School of Natural Sciences, National University of Sciences and Technology, Sector H-12, Islamabad, Pakistan \\ Correspondence should be addressed to Umer Saeed; umer.math@gmail.com
}

Received 25 February 2014; Accepted 29 June 2014; Published 15 July 2014

Academic Editor: Marianna A. Shubov

Copyright (C) 2014 U. Saeed and M. ur Rehman. This is an open access article distributed under the Creative Commons Attribution License, which permits unrestricted use, distribution, and reproduction in any medium, provided the original work is properly cited.

We utilized the Haar wavelet operational matrix method for fractional order nonlinear oscillation equations and find the solutions of fractional order force-free and forced Duffing-Van der Pol oscillator and higher order fractional Duffing equation on large intervals. The results are compared with the results obtained by the other technique and with exact solution.

\section{Introduction}

Haar wavelet is the lowest member of Daubechies family of wavelets and is convenient for computer implementations due to availability of explicit expression for the Haar scaling and wavelet functions [1]. Operational approach is pioneered by Chen and Hsiao [2] for uniform grids. The basic idea of Haar wavelet technique is to convert differential equations into a system of algebraic equations of finite variables. The Haar wavelet technique for solving linear homogeneous/inhomogeneous, constant, and variable coefficients has been discussed in [3].

The fractional order forced Duffing-Van der Pol oscillator is given by the following second order differential equation [4]:

$$
\begin{gathered}
{ }^{c} D^{\alpha} y(t)-\mu\left(1-y^{2}(t)\right) y^{\prime}(t)+a y(t)+b y^{3}(t) \\
=g(f, \omega, t), \quad 1<\alpha \leq 2,
\end{gathered}
$$

where ${ }^{c} D^{\alpha}$ is the Caputo derivative; $g(f, \omega, t)=f \cos (\omega t)$ represents the periodic driving function of time with period $T=2 \pi / \omega$, where $\omega$ is the angular frequency of the driving force; $f$ is the forcing strength; and $\mu>0$ is the damping parameter of the system. Duffing-Van der Pol oscillator equation can be expressed in three physical situations:
(1) single-well $a>0, b>0$;

(2) double-well $a<0, b>0$;

(3) double-hump $a>0, b<0$.

The quasilinearization approach was introduced by Bellman and Kalaba $[5,6]$ as a generalization of the NewtonRaphson method [7] to solve the individual or systems of nonlinear ordinary and partial differential equations. The quasilinearization approach is suitable to general nonlinear ordinary or partial differential equations of any order.

The Haar wavelets with quasilinearization technique [810] are applied for the approximate solution of integer order nonlinear differential equations. In [11], we extend the Haar wavelet - quasilinearization technique for fractional nonlinear differential equations.

The aim of the present work is to investigate the solution of the higher order fractional Duffing equation, fractional order force-free and forced Duffing-Van der pol (DVP) oscillator using Haar wavelet-quasilinearization technique. We have discussed the three special situations of DVP oscillator equation such as single-well, double-well, and double- hump.

\section{Preliminaries}

In this section, we review basic definitions of fractional differentiation and fractional integration [12]. 
(1) Riemann-Liouville fractional integral operator of order $\alpha$ is as follows:

the Riemann-Liouville fractional order integral of order $\alpha \in \mathbb{R}^{+}$is defined as

$$
I_{x}^{\alpha} y(x)=\frac{1}{\Gamma(\alpha)} \int_{a}^{x}(x-t)^{\alpha-1} y(t) d t,
$$

for $a<x \leq b$.

(2) Riemann-Liouville and Caputo fractional derivative operators of order $\alpha$ are as follows:

the Riemann-Liouville fractional order derivative of order $\alpha \in \mathbb{R}^{+}$is defined as

$$
{ }^{r} D_{x}^{\alpha} y(x)=\frac{1}{\Gamma(n-\alpha)}\left(\frac{d}{d x}\right)^{n} \int_{a}^{x}(x-t)^{n-\alpha-1} y(t) d t,
$$

for $a<x \leq b$, where $n-1<\alpha<n, n \in \mathbb{N}$, and $n=\lceil\alpha\rceil$.

The Caputo fractional order derivative of order $\alpha \in \mathbb{R}^{+}$is defined as

$$
{ }^{c} D_{x}^{\alpha} y(x)=\frac{1}{\Gamma(n-\alpha)} \int_{a}^{x}(x-t)^{n-\alpha-1}\left(\frac{d}{d t}\right)^{n} y(t) d t,
$$

for $a<x \leq b$, where $n-1<\alpha<n, n \in \mathbb{N}$, and $n=\lceil\alpha\rceil$.

\section{The Haar Wavelets}

The Haar functions contain just one wavelet during some subinterval of time and remain zero elsewhere and are orthogonal. The $i$ th uniform Haar wavelet $h_{i}(x), x \in[a, b]$ is defined as [2]

$$
h_{i}(x)= \begin{cases}1, & a+(b-a) \frac{k}{m} \leq x<a+(b-a) \frac{k+0.5}{m} ; \\ -1, & a+(b-a) \frac{k+0.5}{m} \leq x<a+(b-a) \frac{k+1}{m} \\ 0, & \text { otherwise, }\end{cases}
$$

where $i=2^{j}+k+1 ; j=0,1,2, \ldots, J$ is dilation parameter, where $m=2^{j}$ and $k=0,1,2, \ldots, 2^{j}-1$ is translation parameter. $J$ is maximal level of resolution and the maximal value of $i$ is $2 M$ where $M=2^{J}$. In particular $h_{1}(x):=\chi_{[a, b]}(x)$, where $\chi_{[a, b]}(x)$ is characteristic function on interval $[a, b]$, is the Haar scaling function. For the uniform Haar wavelet, the wavelet-collocation method is applied. The collocation points for the uniform Haar wavelets are usually taken as $x_{j}=a+(b-a)((j-0.5) / 2 M)$, where $j=1,2, \ldots, 2 M$.

3.1. Fractional Integral of the Haar Wavelets. Any function $y \in L_{2}[a, b]$ can be represented in terms of the uniform Haar series

$$
\begin{aligned}
& y(x)=\sum_{l=1}^{2 M} b_{l} h_{l}(x), \quad l=2^{j}+k+1, \\
& j=0,1,2, \ldots, J, \quad k=0,1,2, \ldots, 2^{j}-1,
\end{aligned}
$$

where $b_{l}$ are the Haar wavelet coefficients given as $b_{l}=$ $\int_{-\infty}^{\infty} y(x) h_{l}(x) d x$.

Any function of two variables $u(x, t) \in L_{2}[a, b] \times[a, b]$ can be approximated as

$$
u(x, t) \approx \sum_{l=1}^{2 M} \sum_{i=1}^{2 M} c_{l, i} h_{l}(x) h_{i}(t)=H^{T}(x) C H(t),
$$

where $C$ is $2 M \times 2 M$ coefficient matrix which can be determined by the inner product $c_{l, i}=\left\langle h_{l}(x),\left\langle u(x, t), h_{i}(t)\right\rangle\right\rangle$.

The Riemann-Liouville fractional integral of the uniform Haar wavelets is given as

$$
\begin{gathered}
I_{x}^{\alpha} h_{1}(x)=\frac{(x-a)^{\alpha}}{\Gamma(\alpha+1)}, \\
P_{\alpha, l}(x)=I_{x}^{\alpha} h_{l}(x)=\frac{1}{\Gamma(\alpha)} \int_{a}^{x}(x-s)^{\alpha-1} h_{l}(s) d s \\
=\frac{1}{\Gamma(\alpha+1)}\left\{\begin{array}{c}
(x-a(l))^{\alpha}, \\
a(l) \leq x<b(l) ; \\
(x-a(l))^{\alpha}-2(x-b(l))^{\alpha}, \\
b(l) \leq x<c(l) ; \\
(x-a(l))^{\alpha}-2(x-b(l))^{\alpha}+(x-c(l))^{\alpha}, \\
x \geq c(l),
\end{array}\right.
\end{gathered}
$$

where $a(l)=a+(b-a)(k / m), b(l)=a+(b-a)((k+0.5) / m)$, and $c(l)=a+(b-a)((k+1) / m)$.

\section{Convergence Analysis}

Our work is based on quasilinearization technique and Haar wavelet method; first, we analyze the convergence of both schemes and then describe the role of their convergence according to present work.

4.1. Convergence of Quasilinearization Technique [6]. Consider the nonlinear second order differential equation:

$$
y^{\prime \prime}(x)=f(y), \quad y(0)=y(b)=0 .
$$

Applying quasilinearization technique to (10) yields

$$
\begin{aligned}
& y_{n+1}^{\prime \prime}(x)=f\left(y_{n}\right)+\left(y_{n+1}-y_{n}\right) f^{\prime}\left(y_{n}\right), \\
& y_{n+1}(0)=y_{n+1}(b)=0 .
\end{aligned}
$$

Let $y_{0}(x)$ be some initial approximation. Each function $y_{n+1}(x)$ is a solution of a linear equation (11), where $y_{n}$ is always considered to be known and is obtained from the previous iteration.

According to [6] and letting $\max _{y}\left(|f(y)|,\left|f^{\prime}(y)\right|\right)=m<$ $\infty$ and $k=\max _{u}\left|f^{\prime \prime}(u)\right|$, we have

$$
\max _{x}\left|y_{n+1}-y_{n}\right| \leq \frac{b^{2}(k / 8)}{1-\left(b^{2} m / 4\right)}\left(\max _{x}\left(\left|y_{n}-y_{n-1}\right|\right)^{2} .\right.
$$

This shows that quasilinearization technique has quadratic convergence, if there is convergence at all. 
4.2. Convergence of Haar Wavelet Method [15]. Let $y(x)$ be a differentiable function and assume that $y(x)$ have bounded first derivative on $(0,1)$; that is, there exist $K>0$; for all $x \in$ $(0,1)$

$$
\left|y^{\prime}(x)\right| \leq K
$$

Haar wavelet approximation for the function $y(x)$ is given by

$$
y_{M}(x)=\sum_{l=1}^{2 M} b_{l} h_{l}(x)
$$

Babolian and Shahsavaran [15] gave $L_{2}$-error norm for Haar wavelet approximation, which is

$$
\left\|y(x)-y_{M}(x)\right\|^{2} \leq \frac{K^{2}}{3} \cdot \frac{1}{(2 M)^{2}}
$$

or

$$
\left\|y(x)-y_{M}(x)\right\| \leq O\left(\frac{1}{M}\right)
$$

As $M=2^{J}$ and $J$ is the maximal level of resolution, according to (16), we conclude that error is inversely proportional to the level of resolution. Equation (16) ensures the convergence of Haar wavelet approximation at higher level of resolution, that is, when $M$ is increased.

Each iteration of quasilinearization technique gives linear differential equation in $y_{n+1}(x)$ which is solved to get approximate value of $y_{n+1}(x), y_{n+1, M}(x)$, by Haar wavelet method. Since solution of our problems has bounded first derivatives over $(0,1)$, according to $(16), y_{n+1, M}(x)$ converges fast to $y_{n+1}(x)$ if we consider the higher level of resolution $J$; that is, we get more accurate results while increasing $J$, and at the same time quasilinearization technique works; that is, given an initial approximation $y_{0}(x)$, we get solution $y_{1}(x)$ of linear differential equation (11) by Haar wavelet method and at next iteration, we get $y_{2}(x)$ and so on. Since quasilinearization technique is second order accurate so it gives rapid convergence, if there is convergence at all. We conclude that solution by Haar wavelet quasilinearization technique $y_{n+1, M}(x)$ converges to exact solution $y(x)$ when both $J$ and $n$ approach $\infty$.

\section{Applications}

In this section, we solve force-free Duffing-Van der Pol oscillator of fractional order, forced Duffing-Van der Pol oscillator of fractional order, and higher order fractional Duffing equation by the Haar wavelet-quasilinearization technique and compare the results with those obtained by other methods and exact solution.

\subsection{Forced Duffing-Van Der Pol Oscillator Equation [4]}

Example 1. Consider the $\alpha$ th order fractional forced DVP oscillator equation

$$
\begin{gathered}
{ }^{c} D^{\alpha} y(x)-\mu\left(1-y^{2}(x)\right) y^{\prime}(x)+a y(x)+b y^{3}(x) \\
=f \cos (\omega x), \quad 1<\alpha \leq 2,
\end{gathered}
$$

subject to the initial conditions $y(0)=1$ and $y^{\prime}(0)=0$. obtain

Applying the quasilinearization technique to (17), we

$$
\begin{aligned}
{ }^{c} D^{\alpha} y_{n+1}(x)-\mu\left(1-y_{n}^{2}(x)\right) y_{n+1}^{\prime}(x) \\
+\left(a+2 \mu y_{n}(x) y_{n}^{\prime}(x)+3 b y_{n}^{2}(x)\right) y_{n+1}(t) \\
=f \cos (\omega x)+2 \mu y_{n}^{2}(x) y_{n}^{\prime}(x)+2 b y_{n}^{3}(x),
\end{aligned}
$$

with the initial conditions $y_{n+1}(0)=1$ and $y_{n+1}^{\prime}(0)=0$.

Now we apply the Haar wavelet method to (18) and approximate the higher order derivative term by the Haar wavelet series as

$$
{ }^{c} D^{\alpha} y_{n+1}(x)=\sum_{l=1}^{2 M} b_{l} h_{l}(x)
$$

Lower order derivatives are obtained by integrating (19) and use the initial condition

$$
y_{n+1}(x)=\sum_{l=1}^{2 M} b_{l} p_{\alpha, l}(x)+1, \quad y_{n+1}^{\prime}(x)=\sum_{l=1}^{2 M} b_{l} p_{\alpha-1, l}(x)
$$

Substitute (19) and (20) into (18) to get

$$
\begin{aligned}
\sum_{l=1}^{2 M} b_{l}\left[h_{l}(x)-\mu\left(1-y_{n}^{2}(x)\right) p_{\alpha-1, l}(x)\right. \\
\left.+\left(a+2 \mu y_{n}(x) y_{n}^{\prime}(x)+3 b y_{n}^{2}(x)\right) p_{\alpha, l}(x)\right] \\
=f \cos (\omega x)+2 \mu y_{n}^{2}(x) y_{n}^{\prime}(x)+2 b y_{n}^{3}(x) \\
-\left(a+2 \mu y_{n}(x) y_{n}^{\prime}(x)+3 b y_{n}^{2}(x)\right)
\end{aligned}
$$

with the initial approximations $y_{0}(x)=1$ and $y_{0}^{\prime}(x)=0$.

(1) (Single-well $a>0, b>0) . a=0.5, b=0.5, \mu=0.1$, $f=0.5, \omega=0.79$.

(2) (Double-well $a<0, b>0$ ). $a=-0.5, b=0.5, \mu=0.1$, $f=0.5, \omega=0.79$.

(3) (Double-hump $a>0, b<0$ ). $a=0.5, b=-0.5$, $\mu=0.1, f=0.5, \omega=0.79$.

The results obtained using the Haar wavelet quasilinearization technique at fifth iteration for the three situations, single-well, double-well, and double-hump, are given in Tables 1, 2, and 3, respectively. Here, we fix the order of equation, $\alpha=2$, and level of resolution $J=9$. We compared the obtained solution with variational iteration method [13], homotopy perturbation method [13], and numerical solution based on the fourth-order Runge-Kutta (RK) method. Also the absolute error relative to RK method is shown in Tables 1,2 , and 3 . It shows that obtained results are more accurate as compared to variational iteration method and homotopy perturbation method. Figures 1, 2, and 3 showed the solution 
TABLE 1: Single-well situation: comparison of solutions by the Haar wavelet-quasilinearization technique $y_{\mathrm{HAAR}}$ at 5 th iteration and level of resolutions $J=9$ with numerical methods [13] and numerical solution based on the fourth-order Runge-Kutta.

\begin{tabular}{lccccc}
\hline \multicolumn{5}{c}{$\alpha=2$} \\
\hline 5th iteration & \multicolumn{5}{c}{$J=9$} \\
\hline$x$ & $y_{\mathrm{RK}}$ & $y_{\mathrm{HPM}}[13]$ & $y_{\mathrm{VIM}}[13]$ & $y_{\mathrm{HAAR}}$ & $\begin{array}{c}\text { Absolute } \\
\text { Error }\end{array}$ \\
\hline 0.2 & 0.9900451 & 0.99004 & 0.99004 & 0.9900451 & $3.1 e-8$ \\
0.4 & 0.9607026 & 0.96075 & 0.9607 & 0.9607024 & $1.5 e-7$ \\
0.6 & 0.9134154 & 0.91383 & 0.91341 & 0.9134150 & $3.5 e-7$ \\
0.8 & 0.8502496 & 0.85216 & 0.85025 & 0.8502491 & $5.8 e-7$ \\
1.0 & 0.773523 & 0.77973 & 0.77353 & 0.773522 & $8.0 e-7$ \\
\hline
\end{tabular}

TABLE 2: Double-well situation: comparison of solutions by the Haar wavelet-quasilinearization technique $y_{\mathrm{HAAR}}$ at 5 th iteration and level of resolutions $J=9$ with numerical methods [13] and numerical solution based on the fourth-order Runge-Kutta.

\begin{tabular}{lccccc}
\hline \multicolumn{6}{c}{$\alpha=2$} \\
\hline 5th iteration & \multicolumn{5}{c}{$J=9$} \\
\hline$x$ & $y_{\mathrm{RK}}$ & $y_{\mathrm{VIM}}[13]$ & $y_{\mathrm{HPM}}[13]$ & $y_{\mathrm{HAAR}}$ & $\begin{array}{c}\text { Absolute } \\
\text { Error }\end{array}$ \\
\hline 0.2 & 1.009945 & 1.00994 & 1.00994 & 1.009945 & $9.8 e-9$ \\
0.4 & 1.039114 & 1.03911 & 1.03918 & 1.039114 & $6.7 e-8$ \\
0.6 & 1.085448 & 1.08544 & 1.08621 & 1.085448 & $1.9 e-7$ \\
0.8 & 1.145384 & 1.14539 & 1.14937 & 1.145384 & $3.9 e-7$ \\
1.0 & 1.213777 & 1.21382 & 1.22785 & 1.213778 & $6.4 e-7$ \\
\hline
\end{tabular}

TABLE 3: Double-hump situation: comparison of solutions by the Haar wavelet-quasilinearization technique $y_{\text {HAAR }}$ at 5 th iteration and level of resolutions $J=9$ with numerical methods [13] and numerical solution based on the fourth-order Runge-Kutta.

\begin{tabular}{lccccc}
\hline \multicolumn{6}{c}{$\alpha=2$} \\
\hline 5th iteration & \multicolumn{5}{c}{$J=9$} \\
\hline$x$ & $y_{\mathrm{RK}}$ & $y_{\mathrm{VIM}}[13]$ & $y_{\mathrm{HPM}}[13]$ & $y_{\mathrm{HAAR}}$ & $\begin{array}{c}\text { Absolute } \\
\text { Error }\end{array}$ \\
\hline 0.1 & 1.00250 & 1.0025 & 1.0025 & 1.00250 & $2.5 e-9$ \\
0.2 & 1.01001 & 1.01001 & 1.01001 & 1.01001 & $4.3 e-11$ \\
0.5 & 1.06301 & 1.063 & 1.06296 & 1.06301 & $4.3 e-8$ \\
0.75 & 1.14347 & 1.14346 & 1.14209 & 1.14347 & $9.8 e-8$ \\
1.0 & 1.26039 & 1.26035 & 1.25055 & 1.26039 & $3.9 e-7$ \\
\hline
\end{tabular}

of (17) for single-well, double-well, and double-hump situations, respectively. We plot the solutions at different order $\alpha$ of (17). Here we fixed the solution at fifth iteration and level of resolution $J=5$ or $J=6$. Also solution by the fourthorder Runge-Kutta method (RK Solution) at $\alpha=2$ is also plotted along with the solution obtained by the Haar wavelet quasilinearization technique (HAAR Solution) and Figures 1, 2 , and 3 show that Haar solution converges to the RK solution when $\alpha$ approaches 2 .

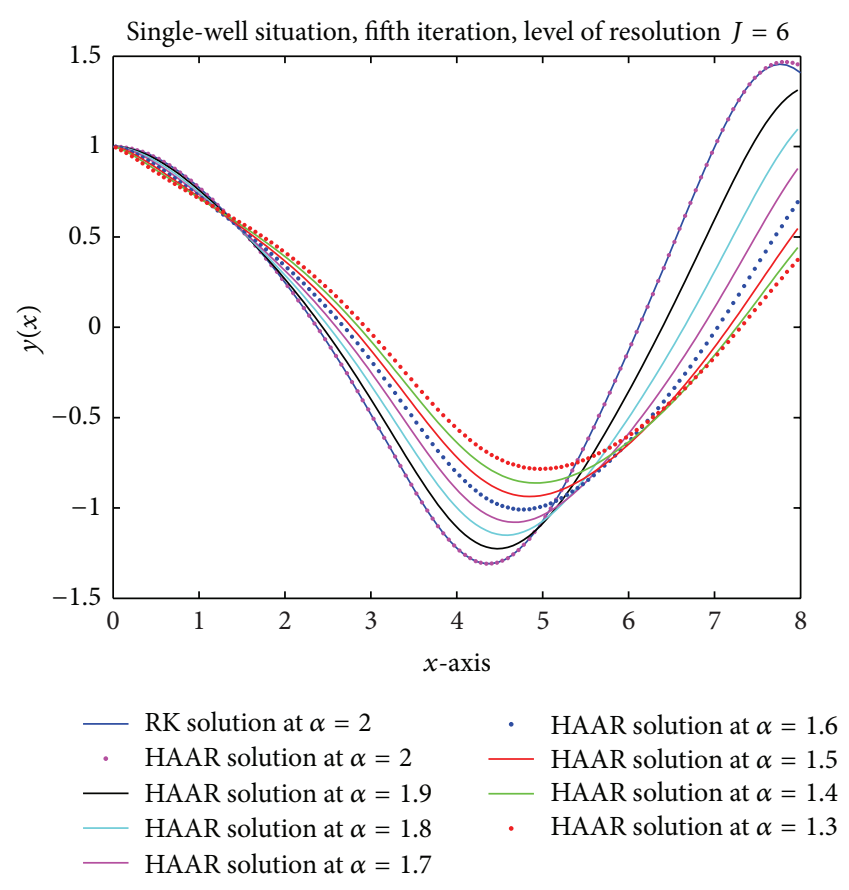

FIgURE 1: Solution by RK method (RK Solution) at $\alpha=2$ and solution by Haar wavelet-quasilinearization technique (HAAR Solution) at $J=6$ and different values of $\alpha$ for $a=0.5, b=0.5$, $\mu=0.1, f=0.5$, and $\omega=0.79$.

\subsection{Force-Free Duffing-Van der Pol Oscillator Equation [16]}

Example 2. Consider the $\alpha$ th order fractional force-free DVP oscillator equation

$$
\begin{array}{r}
{ }^{c} D^{\alpha} y(x)-\mu\left(1-y^{2}(x)\right) y^{\prime}(x)+a y(x)+b y^{3}(x)=0, \\
1<\alpha \leq 2,
\end{array}
$$

subject to the initial conditions $y(0)=1$ and $y^{\prime}(0)=0$. gives

The Haar wavelet-quasilinearization technique on (22)

$$
\begin{aligned}
\sum_{l=1}^{2 M} b_{l}\left[h_{l}(x)-\mu\left(1-y_{n}^{2}(x)\right) p_{\alpha-1, l}(x)\right. \\
\left.\quad+\left(a+2 \mu y_{n}(x) y_{n}^{\prime}(x)+3 b y_{n}^{2}(x)\right) p_{\alpha, l}(x)\right] \\
=2 \mu y_{n}^{2}(x) y_{n}^{\prime}(x)+2 b y_{n}^{3}(x) \\
\quad-\left(a+2 \mu y_{n}(x) y_{n}^{\prime}(x)+3 b y_{n}^{2}(x)\right),
\end{aligned}
$$

with the initial approximations $y_{0}(x)=1$ and $y_{0}^{\prime}(x)=0$.

Results of fifth iteration by the Haar wavelet quasilinearization technique at fixed level of resolution $J=9$ and at $\alpha=2$ are shown in Table 4 . Here we consider $\mu=0.1$, $a=1$, and $b=0.01$ and compare the obtained solution with Adomian decomposition method [16]. Equation (22) is also solved by the fourth-order Runge-Kutta method to 


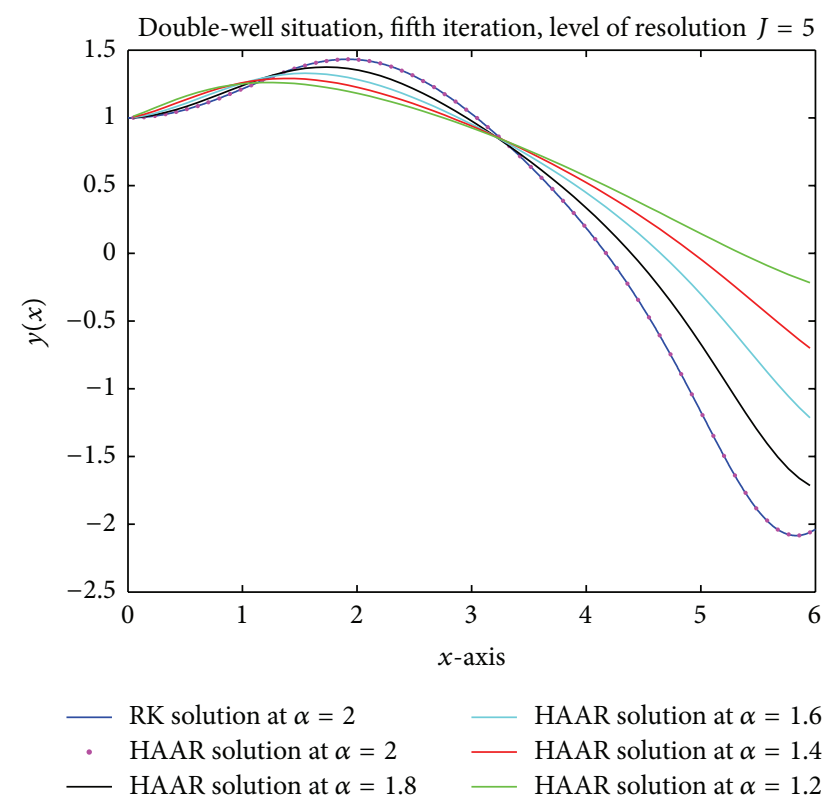

FIGURE 2: Solution by RK method (RK Solution) at $\alpha=2$ and solution by Haar wavelet-quasilinearization technique (HAAR Solution) at $J=5$ and different values of $\alpha$ for $a=-0.5, b=0.5$, $\mu=0.1, f=0.5$, and $\omega=0.79$.

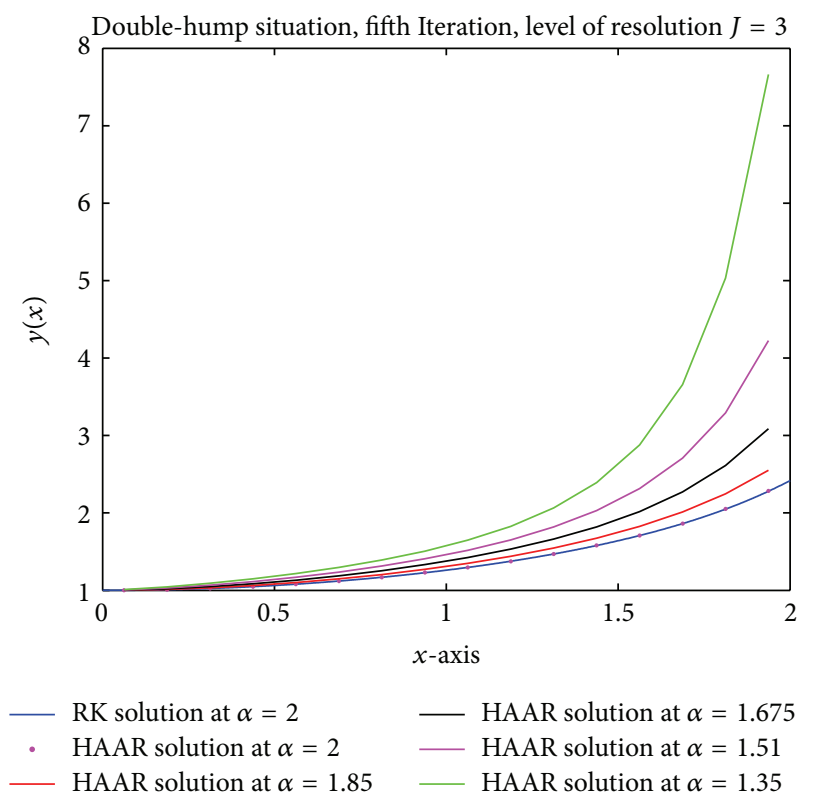

FIGURE 3: Solution by RK method (RK Solution) at $\alpha=2$ and solution by Haar wavelet-quasilinearization technique (HAAR Solution) at $J=5$ and different values of $\alpha$ for $a=0.5, b=-0.5$, $\mu=0.1, f=0.5$, and $\omega=0.79$.

show the applicability of the Haar wavelet quasilinearization technique. Table 4 shows that solution by the Haar wavelet quasilinearization technique gives more accurate results as compared to Adomian decomposition method.

Results of fifth iteration by the Haar wavelet quasilinearization technique at fixed level of resolution $J=5$ and
TABle 4: Force-free Duffing-Van der Pol Oscillator Equation: comparison of solutions by the Haar wavelet-quasilinearization technique $y_{\mathrm{HAAR}}$ at 5 th iteration and level of resolutions $J=9$ with decomposition method $y_{\mathrm{ADM}}[13]$ and numerical solution based on the fourth-order Runge-Kutta.

\begin{tabular}{lcccc}
\hline \multicolumn{5}{c}{$\alpha=2$} \\
\hline 5th iteration & & & $J=9$ & \\
\hline$x$ & $y_{\mathrm{RK}}$ & $y_{\mathrm{ADM}}[13]$ & $y_{\mathrm{HAAR}}$ & $\begin{array}{c}\text { Absolute } \\
\text { Error }\end{array}$ \\
\hline 0.0 & 2.00000 & 1.99750 & 2.00000 & $2.1 e-12$ \\
0.1 & 1.98971 & 1.98724 & 1.98971 & $1.7 e-7$ \\
0.2 & 1.95936 & 1.95697 & 1.95936 & $3.5 e-7$ \\
0.3 & 1.90980 & 1.90758 & 1.90980 & $5.4 e-7$ \\
0.4 & 1.84202 & 1.84008 & 1.84202 & $7.3 e-7$ \\
0.5 & 1.75702 & 1.75552 & 1.75702 & $9.2 e-7$ \\
0.6 & 1.65586 & 1.65493 & 1.65586 & $1.1 e-6$ \\
0.7 & 1.53958 & 1.53937 & 1.53958 & $1.3 e-6$ \\
0.8 & 1.40923 & 1.53937 & 1.40923 & $1.4 e-6$ \\
0.9 & 1.26586 & 1.26726 & 1.26586 & $1.6 e-6$ \\
1.0 & 1.11054 & 1.11267 & 1.11054 & $1.7 e-6$ \\
1.1 & 0.94435 & 0.94704 & 0.94435 & $1.9 e-6$ \\
1.2 & 0.76846 & 0.77147 & 0.76846 & $2.0 e-6$ \\
1.3 & 0.58411 & 0.58715 & 0.58410 & $2.1 e-6$ \\
1.4 & 0.39267 & 0.39545 & 0.39267 & $2.3 e-6$ \\
1.5 & 0.19567 & 0.19795 & 0.19566 & $2.4 e-6$ \\
\hline
\end{tabular}

at different values of $\alpha$ are shown in Figure 4, along with the $\mathrm{RK}$ solution at $\alpha=2$. Figure 4 showed that obtained solution converges to the RK solution when $\alpha$ approaches 2 .

\subsection{Higher Order Oscillation Equation [14]}

Example 3. Consider the $\alpha$ th order fractional Duffing equation

$$
{ }^{c} D^{\alpha} y(x)+5 y^{\prime \prime}(x)+4 y(x)-\frac{1}{6} y^{3}(x)=0, \quad 3<\alpha \leq 4,
$$

subject to the initial conditions:

$$
\begin{aligned}
& y(0)=0, \quad y^{\prime}(0)=1.91103, \\
& y^{\prime \prime}(0)=0, \quad y^{\prime \prime \prime}(0)=-1.15874 .
\end{aligned}
$$

The exact solution, when $\alpha=4$, is given by

$$
\begin{aligned}
y(x)= & 2.1906 \sin (0.9 x)-0.02247 \sin (2.7 x) \\
& +0.000045 \sin (4.5 x) .
\end{aligned}
$$

Quasilinearization technique to (24) gives

$$
\begin{gathered}
{ }^{c} D^{\alpha} y_{n+1}(x)+5 y_{n+1}^{\prime \prime}(x)+\left(4-\frac{1}{2} y_{n}^{2}(x)\right) y_{n+1}(x) \\
=-\frac{1}{3} y_{n}^{3}(x), \quad 3<\alpha \leq 4,
\end{gathered}
$$




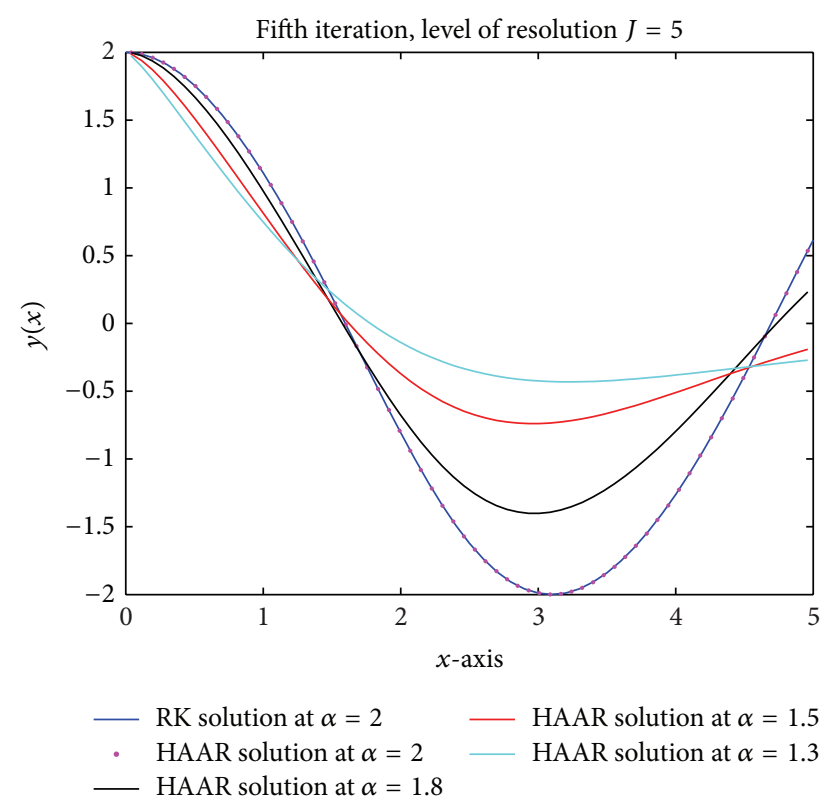

FIGURE 4: Solution by RK method (RK Solution) at $\alpha=2$ and solution by Haar wavelet-quasilinearization technique (HAAR Solution) at $J=5$ and different values of $\alpha$ for $a=1, \mu=0.1$, $b=0.01$, and $f=0$.

with the initial conditions:

$$
\begin{array}{ll}
y_{n+1}(0)=0, & y_{n+1}^{\prime}(0)=1.91103 \\
y_{n+1}^{\prime \prime}(0)=0, & y_{n+1}^{\prime \prime \prime}(0)=-1.15874 .
\end{array}
$$

Implement the Haar wavelet method to (27) as follows:

$$
{ }^{c} D^{\alpha} y_{n+1}(x)=\sum_{l=1}^{2 M} b_{l} h_{l}(x)
$$

Lower order derivatives are obtained by integrating (29) and use the initial condition

$$
\begin{aligned}
& y_{n+1}(x)=\sum_{l=1}^{2 M} b_{l} p_{\alpha, l}(x)-\frac{1.15874}{6} x^{3}+1.91103 x, \\
& y_{n+1}^{\prime}(x)=\sum_{l=1}^{2 M} b_{l} p_{\alpha-1, l}(x)-\frac{1.15874}{2} x^{2}+1.91103, \\
& y_{n+1}^{\prime \prime}(x)=\sum_{l=1}^{2 M} b_{l} p_{\alpha-2, l}(x)-1.15874 x, \\
& y_{n+1}^{\prime \prime \prime}(x)=\sum_{l=1}^{2 M} b_{l} p_{\alpha-3, l}(x)-1.15874 .
\end{aligned}
$$

TABLE 5: Higher order oscillation equation: comparison of solutions by the Haar wavelet-quasilinearization technique at 6 th iteration and level of resolutions $J=10$ with generalized differential quadrature rule (GDQR) method [14] and exact solution.

\begin{tabular}{lccccc}
\hline \multicolumn{5}{c}{$\alpha=4$} \\
\hline 6 th iteration & \multicolumn{5}{c}{$J=10$} \\
\hline$x$ & $y_{\text {Exact }}[14]$ & $y_{\text {GDQR }}[14]$ & $E_{\text {GDQR }}[14]$ & $y_{\text {HAAR }}$ & $E_{\text {HAAR }}$ \\
\hline 0.0 & 0 & 0 & 0 & 0 & 0 \\
0.7 & 1.2692 & 1.2693 & -0.002 & 1.2692 & 0.0025 \\
1.4 & 2.0990 & 2.0993 & -0.010 & 2.0990 & 0.0037 \\
2.1 & 2.0929 & 2.0933 & -0.019 & 2.0928 & 0.0048 \\
2.8 & 1.2541 & 1.2545 & -0.027 & 1.2541 & 0.0059 \\
3.5 & -0.0179 & -0.0177 & 0.813 & -0.0179 & -0.1679 \\
4.2 & -1.2843 & -1.2842 & 0.003 & -1.2843 & -0.0010 \\
4.9 & 1.0880 & -2.1051 & -0.004 & 1.0879 & 0.0063 \\
5.6 & -2.0866 & -2.0868 & -0.014 & -2.0865 & -0.0046 \\
6.3 & -1.2390 & -1.2395 & -0.039 & -1.2389 & -0.0083 \\
7.0 & 0.0357 & 0.0352 & 1.276 & 0.0358 & 0.2095 \\
7.7 & 1.2992 & 1.2990 & 0.013 & 1.2992 & 0.0010 \\
8.4 & 2.1109 & 2.1111 & -0.009 & 2.1108 & 0.0031 \\
9.1 & 2.0801 & 2.0805 & -0.021 & 2.0800 & 0.0056 \\
9.8 & 1.2237 & 1.2243 & -0.044 & 1.2236 & 0.0099 \\
10.5 & -0.0536 & -0.0529 & 1.146 & -0.0537 & -0.1965 \\
11.2 & -1.3141 & -1.3136 & 0.037 & -1.3141 & -0.0042 \\
11.9 & -2.1166 & -2.1166 & -0.002 & -2.1166 & -0.0022 \\
12.6 & -2.0734 & -2.0741 & -0.030 & -2.0733 & -0.0068 \\
13.3 & -1.2084 & -1.2093 & -0.071 & -1.2082 & -0.0136 \\
14.0 & 0.0714 & 0.0706 & 1.057 & 0.0715 & 0.1888 \\
\hline & & & & &
\end{tabular}

Substitute (29) and (30) into (27), we get

$$
\begin{aligned}
\sum_{l=1}^{2 M} b_{l} & {\left[h_{l}(x)+5 p_{\alpha-2, l}(x)+\left(4-\frac{1}{2} y_{n}^{2}(x)\right) p_{\alpha, l}(x)\right] } \\
= & -\frac{1}{3} y_{n}^{3}(x)+5(1.15874) x \\
& -\left(4-\frac{1}{2} y_{n}^{2}(x)\right)\left(1.91103 x-\frac{1.15874}{6} x^{3}\right),
\end{aligned}
$$

with the initial approximations:

$$
\begin{array}{ll}
y_{0}(x)=0, & y_{0}^{\prime}(x)=1.91103, \\
y_{0}^{\prime \prime}(x)=0, & y_{0}^{\prime \prime \prime}(x)=-1.15874 .
\end{array}
$$

Solution by the Haar wavelet quasilinearization technique at 6 th fixed level of resolution $J=10$ and order of (24) $\alpha=4$ is shown in Table 5. It shows that obtained solution is more accurate as compared to generalized differential quadrature rule (GDQR) [14]. $E_{\mathrm{GDQE}}$ and $E_{\mathrm{HAAR}}$ represent the percentage error of generalized differential quadrature rule and the Haar wavelet quasilinearization technique, respectively.

We fix the solutions at fifth iteration, level of resolution $J=5$, and plot the solution at different values of $\alpha$ that are shown in Figure 5 along with the exact solution at $\alpha=4$ 


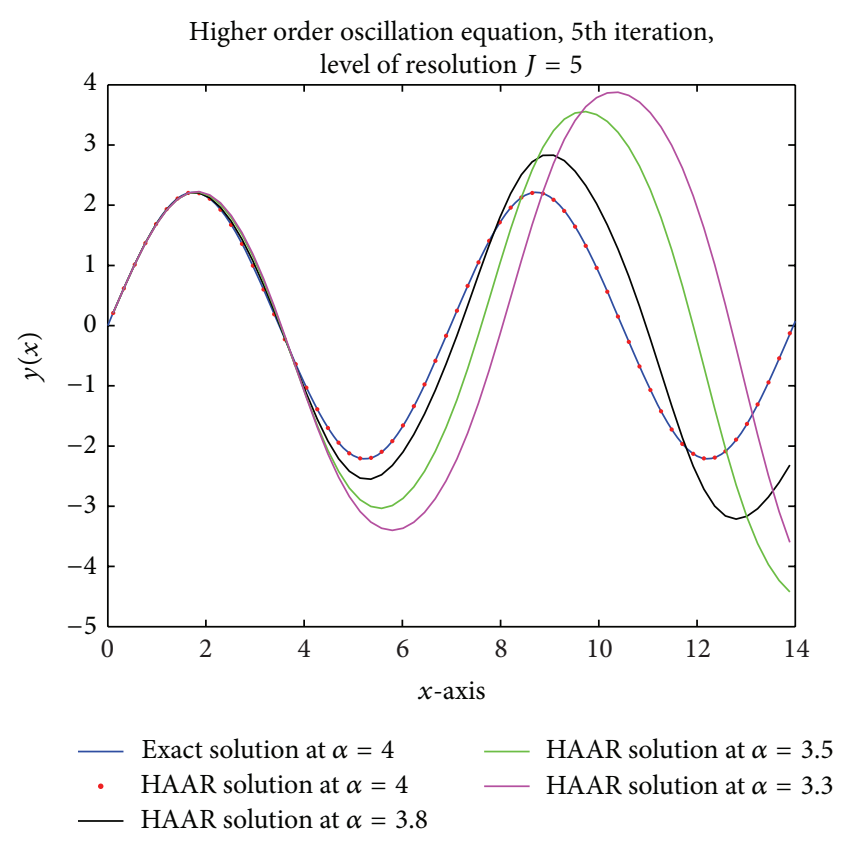

FIgURE 5: Higher order oscillation equation. Exact solution at $\alpha=4$ and solution by Haar wavelet-quasilinearization technique at $J=5$ and different values of $\alpha$.

and Figure 5 shows that solution by the Haar wavelet quasilinearization technique converges to the exact solution, when $\alpha$ approaches 4 .

\section{Conclusion}

It is shown that Haar wavelet method with quasilinearization technique gives excellent results when applied to fractional order nonlinear oscillation equations. The results obtained from Haar wavelet quasilinearization technique are better than the results obtained by other methods and are in good agreement with exact solutions or solution by the fourthorder Runge-Kutta method, as shown in Tables and Figures. The solution of the fractional order nonlinear oscillation equation converges to the solution of integer order nonlinear oscillation differential equation as shown in Figures 1, 2, 3, 4, and 5.

\section{Conflict of Interests}

Umer Saeed and Mujeeb ur Rehman declare that there is no conflict of interests regarding the publication of this paper.

\section{Acknowledgments}

The authors are grateful to the anonymous reviewers for their valuable comments which have led to the improvement of the paper.

\section{References}

[1] I. Daubechies, "The wavelet transform, time-frequency localization and signal analysis," IEEE Transactions on Information Theory, vol. 36, no. 5, pp. 961-1005, 1990.

[2] C. F. Chen and C. H. Hsiao, "Haar wavelet method for solving lumped and distributed-parameter systems," IEE Proceedings Control Theory and Applications, vol. 144, no. 1, pp. 87-94, 1997.

[3] M. Garg and L. Dewan, "A numerical method for linear ordinary differential equatons using non-recursive Haar connection coefficients," International Journal of Computational Science and Mathematics, vol. 2, pp. 429-440, 2010.

[4] S. A. Malik, I. M. Qureshi, M. Zubair, and I. Haq, "Solution to force-free and forced duffing-Van der Pol oscillator using memetic computing," Journal of Basic and Applied Scientific Research, vol. 2, no. 11, pp. 11136-11148, 2012.

[5] R. Kalaba, "On nonlinear differencial equations, the maximum operation and monotone convergence," Journal of Applied Mathematics and Mechanics, vol. 8, pp. 519-574, 1959.

[6] R. E. Bellman and R. E. Kalaba, Quasilinearization and Nonlinear Boundary-Value Problems, Elsevier, New York, NY, USA, 1965.

[7] S. D. Conte and C. de Boor, Elementary Numerical Analysis, McGraw-Hill, New York, NY, USA, 1981.

[8] R. Jiwari, "A Haar wavelet quasilinearization approach for numerical simulation of Burgers' equation," Computer Physics Communications, vol. 183, no. 11, pp. 2413-2423, 2012.

[9] H. Kaur, R. C. Mittal, and V. Mishra, "Haar wavelet quasilinearization approach for solving nonlinear boundary value problems," The American Journal of Computational Mathematics, vol. 1, pp. 176-182, 2011.

[10] H. Kaur, R. C. Mittal, and V. Mishra, "Haar wavelet quasilinearization approach for solving lane emden equations," International Journal of Mathematics and Computer Applications Research, vol. 2, pp. 47-60, 2012.

[11] U. Saeed and M. U. Rehman, "Haar wavelet-quasilinearization technique for fractional nonlinear differential equations," Applied Mathematics and Computation, vol. 220, pp. 630-648, 2013.

[12] C. A. Monje, Y. Chen, B. M. Vinagre, D. Xue, and V. Feliu, Fractional-Order Systems and Controls, Advances in Industrial Control, Springer, London, UK, 2010.

[13] H. Sajadi, D. D. Ganji, and Y. V. Shenas, "Application of numerical and semianalytical approach on Van der PolDuffing oscillators," Journal of Advanced Research in Mechanical Engineering, vol. 1, no. 3, pp. 136-141, 2010.

[14] G. R. Liu and T. Y. Wu, "Numerical solution for differential equations of duffing-type non-linearity using the generalized differential quadrature rule," Journal of Sound and Vibration, vol. 237, no. 5, pp. 805-817, 2000.

[15] E. Babolian and A. Shahsavaran, "Numerical solution of nonlinear Fredholm integral equations of the second kind using Haar wavelets," Journal of Computational and Applied Mathematics, vol. 225, no. 1, pp. 87-95, 2009.

[16] G. Asadi Cordshooli and A. R. Vahidi, "Solutions of Duffingvan der Pol equation using decomposition method," Advanced Studies in Theoretical Physics, vol. 5, no. 1-4, pp. 121-129, 2011. 


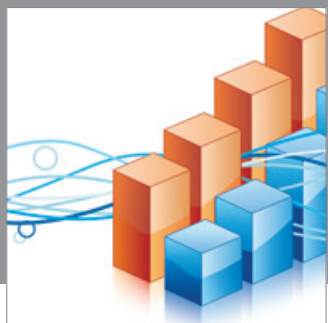

Advances in

Operations Research

mansans

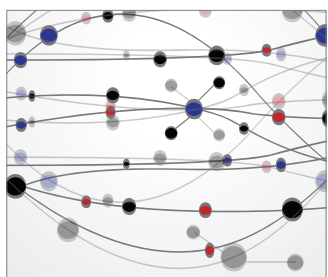

The Scientific World Journal
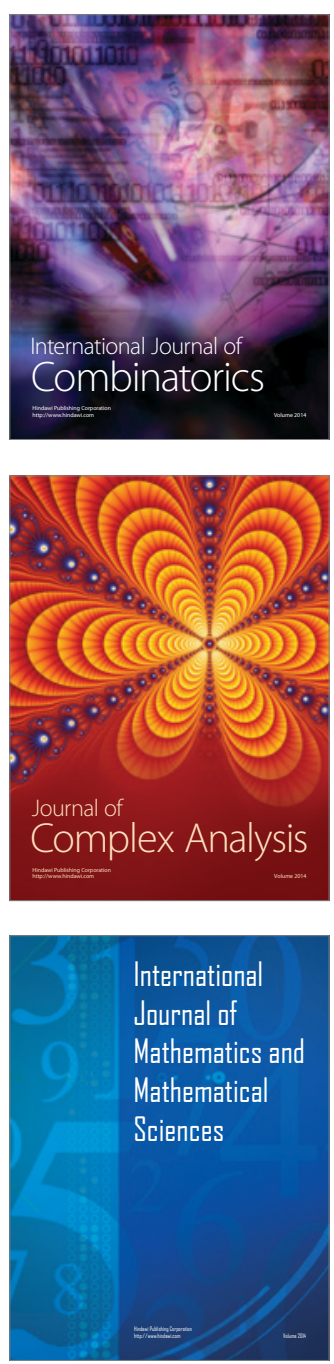
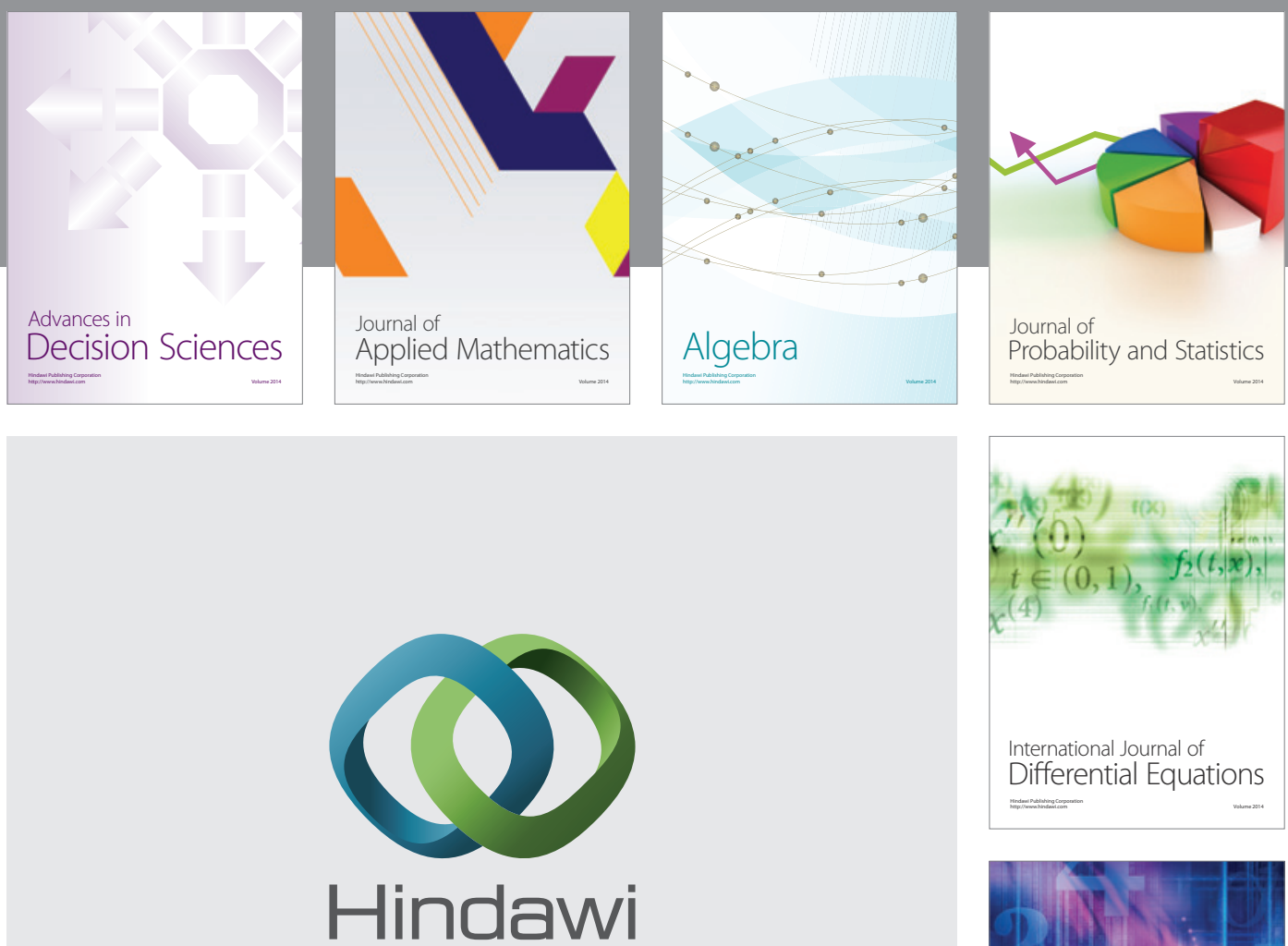

Submit your manuscripts at http://www.hindawi.com
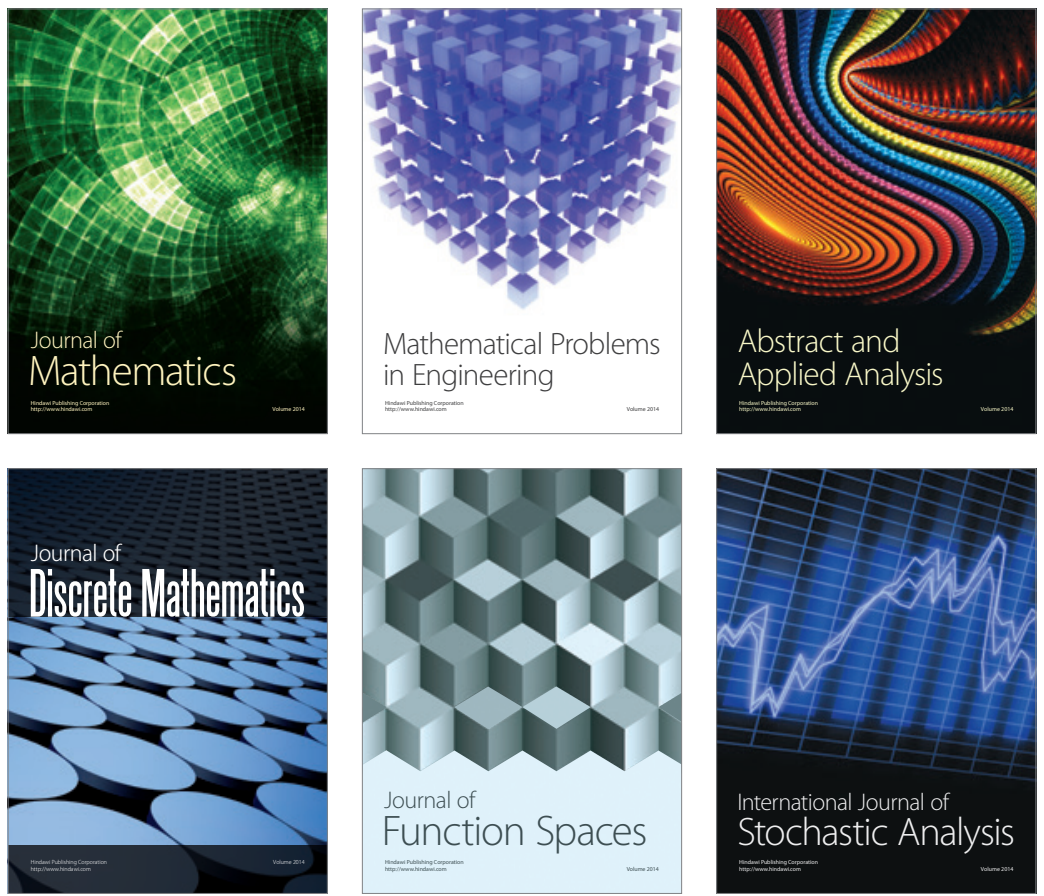

Journal of

Function Spaces

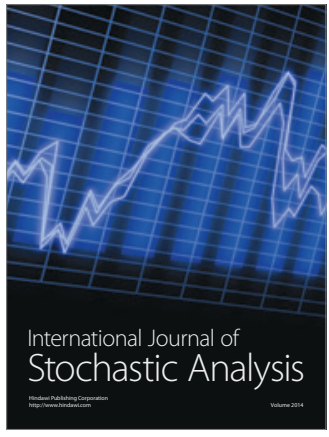

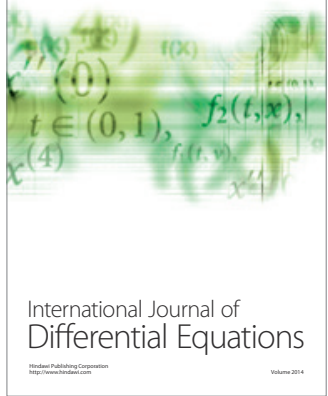
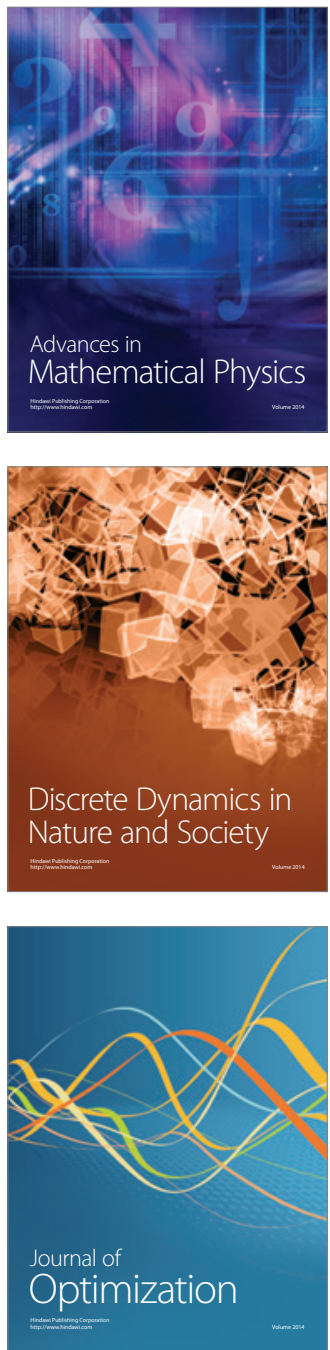